\title{
Controlling Assembly of Colloidal Particles into Structured Objects: Basic Strategy and a Case Study
}

\author{
Michael A. Bevan ${ }^{\mathrm{a}}$, David M. Ford ${ }^{\mathrm{b},{ }^{*}}$, Martha A. Grover ${ }^{\mathrm{c}}$, Benjamin Shapiro ${ }^{\mathrm{d}}$, Dimitrios \\ Maroudas $^{\mathrm{b}}$, Yuguang Yang ${ }^{\mathrm{a}}$, Raghuram Thyagarajan ${ }^{\mathrm{b}}$, Xun Tang ${ }^{\mathrm{c}}$, and Ray M. Sehgal ${ }^{\mathrm{b}}$ \\ ${ }^{a}$ Department of Chemical \& Biomolecular Engineering, Johns Hopkins University \\ ${ }^{\mathrm{b}}$ Department of Chemical Engineering, University of Massachusetts, Amherst \\ ${ }^{\mathrm{c}}$ Department of Chemical \& Biomolecular Engineering, Georgia Institute of Technology \\ ${ }^{\mathrm{d}}$ Department of Bioengineering, University of Maryland
}

to whom correspondence should be addressed, ford@ecs.umass.edu

\begin{abstract}
A computational study is presented in which real-time manipulation of the interaction potential between particles in a colloidal system is used to control their assembly into a close-packed crystalline object. The basic model used throughout the study is a highfidelity representation of a real experimental system in which 32 colloidal silica particles are suspended in aqueous solution with polymer hydrogel providing a temperaturetunable attractive force between the particles. Diffusion mapping is used to determine a set of coarse variables that provide an appropriate low-dimensional representation of this system at four discrete values of the attraction strength. In this case the diffusion mapping process identified two dimensions; one correlates well with the radius of gyration of the entire set of particles and the other correlates well with the average distance between distinct clusters of particles. Two different stochastic models are then built in the two-dimensional (2D) space of these variables, using data from a large number of short Brownian dynamics simulations of the full 32-particle system. The first 2D model is based on a Smoluchowski framework and is used to characterize the overall equilibrium and diffusive properties of the system. The second 2D model is based on a transition rate matrix and is used for process control. A control policy based on an infinite-horizon Markov decision process is developed using the four different attraction strengths as the input variables. The resulting policy is non-trivial; rather than simply selecting the strongest level of attraction, some mix of weak and strong attractions generally provides the optimal approach to the target close-packed state. This study, while focused on the particular mechanism of tunable depletion attraction, suggests a general strategy that could be adapted to different mechanisms of actuating colloidal assembly.
\end{abstract}

Keywords: self-assembly; colloidal particles; diffusion map; Markov decision processes; Smoluchowski equation 


\section{Introduction and Background}

\subsection{Purpose}

The purpose of this paper is to outline a general framework for controlling the selfassembly of a finite collection of colloidal particles into useful structures, and to demonstrate the application of that framework on a simple but physically relevant example.

Prior work in manipulating particle ensembles has largely separated into either selfassembly without real-time feedback control, or feedback control without self-assembly. Self-assembly is a well-studied field documented in multiple review articles. ${ }^{1-3}$ During self-assembly, particle-particle interactions may cause them to assemble into ordered ensembles, and that assembly can be guided by varying external parameters that modulate the strength of interactions, by patterning the substrate on which ensemble formation occurs, or by choosing and/or modifying the properties of the particles. Usually, the number of particles is large and their behavior is most naturally described by statistical models. In contrast, real-time feedback control of micro- and nano-scale particles has been applied to systems with just a few particles whose behavior is modeled deterministically and where control actuation is essentially considered per-particle. Researchers have modulated dielectrophoretic (DEP) forces, ${ }^{4-6}$ optoelectronic actuation, ${ }^{7}$ magnetic fields, ${ }^{8-11}$ and fluid flow and electrokinetic forces ${ }^{12-17}$ to control the position and orientation of one and a few $(<10)$ micro/nano particles and rods. Several approaches have been developed to tune colloidal interactions to mediate crystallization of small ensembles in a reversible manner ${ }^{18-24}$ which are amenable to control via feedback schemes. Our recent work ${ }^{22,25}$ suggests that these two approaches could be combined, and our goal in this paper is to demonstrate that feedback can improve ensemble selfassembly in a high-fidelity model of the assembly process.

\subsection{Colloidal systems}

Colloidal particles with dimensions ranging from nanometers $(\mathrm{nm})$ to microns provide the basic building blocks to control material structures on length scales larger than molecular dimensions but smaller than macroscopically visible dimensions. While standard chemistry deals with building atomic and molecular structures, and microfabrication and granular material processing provide routes toward manipulating miniaturized structures on the order of 10-1000 microns, colloidal assembly provides unique access to material architectures with characteristic dimensions from $1 \mathrm{~nm}$ to 10 microns. Although controlling microstructure is important to determining emergent material properties in any colloidal system (such as in paint and mud), ${ }^{26}$ it becomes increasingly important and justified for creating high value-added materials with exotic electromagnetic properties. In particular, the ability to assemble defect-free colloidal crystals provides a potential scalable manufacturing method to create materials for advanced applications, such as solar-cell coatings, as well as futuristic technologies based on optical computing and cloaking devices. Methods to model and control colloidal crystallization have been developed in recent years. ${ }^{18-22}$ 


\section{Theory}

We first provide some basic physical theory of the thermodynamics and dynamics of colloidal solutions. We take a microscopic approach and describe both the conservative and non-conservative forces that act on the particles, with an emphasis on a certain type of attractive conservative force that is sensitive to temperature. We then discuss how high-dimensional data at the level of the particle coordinates, as generated by simulation (using the forces mentioned above) or experiment, can be used to create low-dimensional stochastic models of the system dynamics. Finally we describe how control of an assembly process can be achieved by using the low-dimensional stochastic models as a real-time guide to manipulating the forces. The flowchart in Fig. 1 provides an overview of the strategy.

\subsection{Basic physics of colloids}

In its simplest form a colloidal solution has two components: the colloid particles and the solvent molecules. There may be more components if the solvent is a mixture of molecular species, or if there is more than one type (or size) of colloid particle. For simplicity the basic two-component case is presented here, and the particle and solvent species are both assumed to be spherical. Relaxation of either of these restrictions poses no additional conceptual difficulty.

Statistical mechanics ${ }^{27}$ provides the basic underlying theory of how the colloid particles and solvent molecules sample positions in space and time, at fixed values of global thermodynamic parameters. There are large differences in size and mass of the two components, as well as the time scales associated with their motion; ${ }^{28}$ this significant asymmetry will allow certain simplifications to be made in the modeling.

The forces acting on the colloid particles are the key elements in a dynamical model. Section 2.1.1 presents a framework for describing the net conservative forces between the particles. Section 2.1.2 presents the important non-conservative (hydrodynamic) forces that arise in colloidal systems. Section 2.1.3 presents a full dynamical model in particle positions and time that incorporates both types of force.

\subsubsection{Conservative forces and interaction potentials in colloidal solutions}

This section is based on a review by Frenkel, ${ }^{29}$ which goes back to the work of Onsager, ${ }^{30}$ and another by Belloni, ${ }^{31}$ which uses McMillan-Mayer solution theory. ${ }^{32,33}$

Assume that a colloidal solution is composed of $N_{c}$ colloid particles, occupying positions $\mathbf{r}^{\left(N_{C}\right)}$, and $N_{s}$ solvent molecules, occupying positions $\mathbf{r}^{\left(N_{S}\right)}$, at a fixed volume $V$ and temperature $T$. The total potential energy may be written as the sum of colloid-colloid (CC), colloid-solvent (CS), and solvent-solvent contributions (SS),

$$
U_{t o t}\left(\mathbf{r}^{\left(N_{C}\right)}, \mathbf{r}^{\left(N_{S}\right)}\right)=U_{C C}\left(\mathbf{r}^{\left(N_{C}\right)}\right)+U_{C S}\left(\mathbf{r}^{\left(N_{C}\right)}, \mathbf{r}^{\left(N_{S}\right)}\right)+U_{S S}\left(\mathbf{r}^{\left(N_{S}\right)}\right) .
$$


(Note that this decomposition does not necessarily imply a pairwise additivity of the potential energy at the particle-molecule level.) The positions of the solvent molecules change much more rapidly than the positions of the colloid particles due to the asymmetry in size, mass, and time scales of the two species. This leads to the reasonable assumption that the positions of the solvent molecules may be averaged over, to obtain an effective potential based on colloid particle positions only. The resulting potential of mean force $W$ has the general form

$$
W\left(\mathbf{r}^{\left(N_{C}\right)}\right)=U_{C C}\left(\mathbf{r}^{\left(N_{C}\right)}\right)-k T \ln \left\langle\exp \left[-U_{C S}\left(\mathbf{r}^{\left(N_{C}\right)}, \mathbf{r}^{\left(N_{S}\right)}\right) / k T\right]\right\rangle_{\mathbf{r}^{\left(N_{S}\right)}},
$$

where $k$ is the Boltzmann constant, $T$ is the temperature, and the brackets denote an average value from Boltzmann sampling over the positions of the solvent molecules. (In fact this sampling is done most conveniently at fixed chemical potential of the solvent $\mu_{s}$ rather than at fixed $N_{s}$. See either Frenkel ${ }^{29}$ or Belloni $^{31}$ for a complete derivation at the level of statistical mechanical partition functions.) The quantity $W$ is not equivalent to $U_{t o t}$; it does not include a solvent-solvent contribution, and the dependence of the colloidsolvent contribution on $\mathbf{r}^{\left(N_{S}\right)}$ has been averaged out. However, $W$ does provide the basis for a complete description of the thermodynamic properties of the colloidal particles in an effective one-component model (OCM) in which they interact through an implicit solvent medium. The force on a given particle $i$ due to these interactions, which is of use when computing dynamic trajectories of particles as discussed in section 2.1.3, is straightforwardly computed from the potential of mean force as

$$
\mathbf{f}_{i}\left(\mathbf{r}^{\left(N_{C}\right)}\right)=-\nabla_{i} W
$$

Predicting or measuring the potential of mean force in a given colloidal solution is not a simple task, even within the OCM. To make the problem more tractable the potential is often treated as pairwise additive, so that

$$
W\left(\mathbf{r}^{\left(N_{C}\right)}\right)=\sum_{i<j} w\left(r_{i j}\right)
$$

where the sum is over all pairs of colloid particles and $r_{i j}=\left|\mathbf{r}_{i}-\mathbf{r}_{j}\right|$ is the center-to-center distance between particles $i$ and $j$. In most pairwise interaction models there are additive contributions representing repulsion and attraction, as

$$
w(r)=w_{r e p}(r)+w_{\text {att }}(r)
$$

The repulsion typically arises from the fact that colloidal particles have some net surface charge in solution due to the dissociation of functional groups and/or preferential adsorption of certain charged species from solution. The basic principle of repulsion 
between like charges then causes repulsion between particles; however, the magnitude and range of this repulsion is modulated by the presence of ions in solution, and the development of accurate models for $w_{\text {rep }}(r)$ has been the subject of much study. ${ }^{31}$ Even in the absence of significant surface charge there will be steric repulsion due to overlaps at the atomic level upon close approach ( $r=2 a$ with $a$ the particle radius) of two particles; this type of repulsion is often modeled in $w_{\text {rep }}(r)$ by a simple hard sphere potential.

An attractive component $w_{a t t}(r)$ in the potential may arise from several different physical phenomena. Dispersion, or van der Waals, attraction is due to interactions between permanent or fluctuating dipoles in the two particles. The combination of these attractive forces with electrostatic repulsion via Eq. (5) yields the well-known Derjaguin, Landau, Verweij, and Overbeek (DLVO) potential, which is a common model for understanding colloidal stability; the attractive forces create an infinitely deep potential well near particle-particle contact $(r=2 a$ ), but the electrostatic repulsion forms a positive potential barrier at intermediate $r$ that reduces the rate at which particle pairs "fall into" the well and irreversibly aggregate. ${ }^{28}$

There are other sources of attractive interactions $w_{\text {att }}(r)$ in colloidal systems. An interesting example, and the one of main interest here, is the introduction of a third component (called the depletant) into the system. The depletant is typically another colloidal species, much smaller in size than the main one, that causes an attraction between the larger particles through an entropic mechanism originally described by Asakura and Oosawa. ${ }^{34}$ Figure $2 \mathrm{a}$ depicts the mechanism. A single isolated large particle excludes the centers of the small particles from a sphere of volume (4/3) $\pi(a+L)^{3}$, where $a$ is the large particle radius and $L$ is the depletant radius. However, when the surface-to-surface separation $(r-2 a)$ of two large particles becomes less than or equal to the diameter $2 L$ of the depletant particle, the excluded volumes of the two spheres partially overlap (red region in Fig. 2a) and the net volume available to the depletant particles is increased by the overlap amount. This effect, which increases the entropy of the total system, is (perhaps surprisingly) strong enough to cause the particles of the main colloidal species to preferentially sample such separations. From the point of view of the colloidal particles, this preferential sampling is equivalent to a pairwise attraction that acts when $r \leq 2(a+L)$. Recently one of our laboratories ${ }^{35,36}$ has implemented an experimental realization of this concept using $\sim 2 \mu \mathrm{m}$ silica particles as the main colloidal species and $200 \mathrm{~nm}$ poly- $N$-isopropylacrylamide (PNIPAM) gel particles as the depletant. They found that the pairwise potential of mean force was modeled well by the traditional Asakura-Oosawa expression

$$
w(r)=w_{r e p}(r)-\Pi\left[\left(\frac{4 \pi}{3}\right)(a+L)^{3}\left(1-\frac{3 r}{4(a+L)}+\frac{r^{3}}{16(a+L)^{3}}\right)\right]
$$

where $w_{\text {rep }}(r)$ is given by a common expression for charge-based repulsion and $\Pi$ is the osmotic pressure of the depletant species. The quantity $\Pi$, which sets the magnitude of the entire depletion-based attraction, is proportional to the volume fraction of solution occupied by the depletant species and therefore proportional to $L^{3}$, the cube of the 
depletant radius. The radius $L$ of the PNIPAM particles is highly sensitive to temperature in the neighborhood of $25^{\circ} \mathrm{C}$, so small changes in temperature can cause large changes in $\Pi$ as shown in Fig. 2b. This type of attraction is interesting for the present contribution because it can be changed over a broad range by using the solution temperature as an input variable. Of course in a real system one must consider issues such as the response time of the system temperature to a change in the desired set point and the role of transient states with temperature gradients; in this paper we consider an idealized model in which system temperature changes are instantaneous and uniform. It should be noted that the potentials in Fig. $2 \mathrm{~b}$ were directly measured using Total Internal Reflection microscopy ${ }^{35,36}$ which can directly measure $k T$-scale and nanometer-scale colloidal interactions $\mathrm{s}^{37,38}$ in contrast to other established force measurement techniques such as the Surface Forces Apparatus ${ }^{39}$ or the Atomic Force Microscope. ${ }^{40}$

\subsubsection{Non-conservative forces and hydrodynamics in colloidal solutions}

The particles also experience non-conservative hydrodynamic and stochastic forces as they move through the solvent. The formalism for modeling these forces ${ }^{41,42}$ starts with the Liouville equation for the probability distribution function of the colloid particles and solvent molecules in phase space, i.e. the complete set of position and momentum coordinates. The large difference in mass between the two species is invoked to justify an integration over the solvent positions and momenta; the result is a Fokker-Planck equation in the phase space of the colloid particles only, with the potential of mean force $W$ describing the conservative forces and a phenomenological friction tensor characterizing the hydrodynamic forces. Furthermore the time scale for momentum relaxation of a colloid particle is assumed to be very short compared to that for position relaxation, so the colloidal particle momentum variables may also be eliminated yielding the Fokker-Planck equation,

$$
\frac{\partial p}{\partial t}=\nabla \cdot \mathbf{D}_{\text {diff }} \cdot\left(\nabla-\frac{1}{k T} \mathbf{F}^{c o n}\right) p
$$

where $p\left(\mathbf{r}^{\left(N_{C}\right)}, t\right)$ is the probability distribution for the positions of the colloidal particles at time $t, \mathbf{F}^{c o n}=-\nabla W$ is the vector of conservative forces acting on the particles, and $\mathbf{D}_{\text {diff }}$ is the diffusion tensor. This specific version of the Fokker-Planck equation, which has the equilibrium solution $p_{e q} \sim \exp (-W / k T)$, is often called the Smoluchowski equation.

The tensor $\mathbf{D}_{\text {diff }}$ captures the hydrodynamic and stochastic forces and is generally not diagonal, as the diffusive motions of the particles are coupled by interactions through the liquid solvent. These off-diagonal hydrodynamic interactions are many-body in nature and can be quite challenging to model in concentrated colloidal solutions. Approximate models, usually involving pairwise additivity at some level, are available and have proven useful. ${ }^{43}$ Hydrodynamic interactions have a much longer range than most pairwise potentials of mean force $w(r)$ and are therefore much more costly to compute. The simplest level of approximation is to completely ignore hydrodynamic coupling between particles but retain the stochastic Brownian forces (arising from collisions with 
the solvent molecules) on each individual particle. In that limit $\mathbf{D}_{\text {diff }}=D_{0} \mathbf{I}$, where $\mathbf{I}$ is the identity matrix and $D_{0}=k T /(6 \pi \eta a)$ with $\eta$ the solvent viscosity.

\subsubsection{Dynamical modeling at the particle level}

2.1.3.1. Theory and numerical simulation. In principle the solution to Eq. (7), $p\left(\mathbf{r}^{\left(N_{C}\right)}, t\right)$, provides the basis for a complete description of the equilibrium and dynamic properties of a system with specified $N_{c}, V, T, W$, and $\mathbf{D}_{\text {diff. }}$ However, solving Eq. (7) directly is not convenient in practice because the partial differential equation has $3 N_{c}$ spatial dimensions. The more common strategy is to perform dynamic simulations of the system using a discrete-time version of the Langevin equation that corresponds to Eq. (7); any desired properties may then be computed as averages over the simulated trajectories. In colloidal systems this approach has been named Stokesian dynamics (SD), in analogy with molecular dynamics (MD) for atomistic systems. ${ }^{43}$ Equation (8) shows the form of a typical SD time-stepper ${ }^{44}$

$$
\mathbf{r}=\mathbf{r}^{0}+\left(\nabla \cdot \mathbf{D}_{\text {diff }}^{0}\right) \Delta t+(k T)^{-1} \mathbf{D}_{\text {diff }}^{0} \cdot\left(\mathbf{F}^{c o n, 0}+\mathbf{F}^{B, 0}\right) \Delta t
$$

where $\Delta t$ is the time interval, the superscript 0 indicates quantities computed at the beginning of the time interval, $\mathbf{r}$ is the vector of particle positions, and $\mathbf{F}^{B}$ is a vector of stochastic forces characterized by a mean $\left\langle\mathbf{F}^{B}\right\rangle=\mathbf{0}$ and a variance $\left\langle\mathbf{F}^{B}(0) \mathbf{F}^{B}(t)\right\rangle=2(k T)^{2} \mathbf{D}_{\text {diff }}^{-1} \delta(t)$, where $\delta(t)$ is the Dirac delta function. As noted in section 2.1.2, $\mathbf{D}_{\text {diff }}$ becomes a diagonal matrix independent of particle positions when the hydrodynamic coupling between particles is neglected; in this limit the second term on the right-had-side of Eq. (8) is zero and SD reduces to the simpler Brownian dynamics (BD). $\mathrm{BD}$ is the technique employed in this paper for all basic particle-level dynamical simulations.

SD time-steppers like Eq. (8) provide the means to carry out a dynamical simulation of the colloidal system at the particle-coordinate level, limited only by the accuracy of the conservative potentials and the hydrodynamic diffusion tensors. However, using SD directly in a process control scheme is not feasible because of the long time required to compute trajectories. Ultimately, one is limited by the maximum allowable time step $\Delta t$ in the SD equation and the speed of the available computer. The former depends somewhat on the nature of the conservative forces in the system but is, typically, on the order of $10^{-4}$ seconds or less. The latter of course depends on the computational resources available. As a concrete example, we observed a roughly 12:1 ratio of computing time to simulated time in our BD simulations of the 32-particle system in this paper, using a single core of an AMD Opteron processor.

2.1.3.2. Verification of simulations against experiments. If we are to build lowdimensional models (section 2.2) based on trajectories from detailed time-steppers like those just described, we must have some level of confidence in the accuracy of the force models employed in the time-steppers. The AO-depletion interaction potential model 
used in this work, Eq. (6), was carefully constructed in previous studies by fitting the model parameters so that BD simulations reproduced certain thermodynamic and transport properties measured in microscopy experiments. Specifically, inverse Monte Carlo methods (including image resolution limiting effects) ${ }^{45-47}$ were used to determine parameters by matching measured and simulated radial distribution functions ${ }^{20}$ and mean square displacement statistics. ${ }^{21}$ We are therefore confident that the BD simulations employed here, using the conservative force model of Eq. (6), provide quite accurate representations of trajectories observed in real laboratory experiments.

\subsection{Dimensionality reduction techniques}

Since controlling colloidal assembly with trajectory data from real-time direct numerical simulation is not feasible (section 2.1.3.1), we pursue the alternative strategy of using such data off-line to build models of reduced dimensionality. We view the problem as consisting of two parts: identifying the minimum dimensionality needed to represent the system and choosing appropriate coordinates for those dimensions, and then building a model in those coordinates. We typically refer to the coordinates as "coarse variables" or "order parameters" (OPs), although they may not necessarily reflect order in the conventional sense.

\subsubsection{Identifying reduced dimensionality and coordinates}

Diffusion map (DMap) analysis, sometimes referred to as the nonlinear analog of principal component analysis, provides a route to identifying low-dimensional representations of a data set. ${ }^{48-50}$ In DMap analysis a Markov matrix is constructed from the data set based on transition probabilities, derived from a chosen distance metric, between points in the set. If the data, comprising points of dimension $n$, are indeed embedded in some lower-dimensional manifold, then the eigenvalue spectrum of the matrix will exhibit a gap after the first $(k+1)$ eigenvalues where $k<n$ (note that the first eigenvalue is trivially 1 for a Markov matrix). The top $k$ non-trivial eigenvectors then provide a mapping to the lower-dimensional space, wherein the $k$ coordinates of a given data point are given by the corresponding entries in these eigenvectors. In past applications to colloidal assembly ${ }^{51-53}$ we have found $k$ values in the range 2-5 for colloidal systems with $n \sim 30-300$ (10-100 particles in 3D), using a Hausdorff distance metric on data sets of $\sim 10^{3}-10^{4}$ snapshots sampled from dynamic trajectories. While DMap analysis does provide a value of $k$ and an accompanying set of reduced-space coordinates for each data point in the set, unfortunately it does not provide an explicit mapping between the $n$-dimensional and $k$-dimensional coordinates. The task of identifying useful OPs in the $n$-dimensional space therefore requires some empiricism.

In the past ${ }^{51-53}$ we have approached this problem by proposing a set of physically meaningful functions of the $n$-dimensional coordinates and evaluating the correlation between their values and the values of the top eigenvectors, across the data set. Here we consider three such functions. The first two, $R_{g}{ }^{*}$ and $\left\langle C_{6}\right\rangle$, have been used in past publications ${ }^{51-53}$ to which we refer the reader for more detail. $R_{g}{ }^{*}$ is the radius of gyration of the particle assembly normalized by the radius of a single particle; it is expected to be a useful metric for discriminating between diffuse and condensed phases. $\left\langle C_{6}\right\rangle$ is the average number of crystalline bonds per particle, with a crystalline bond between two 
particles defined by both spatial proximity and degree of similarity in a sixfold orientational order parameter. As a metric of crystallinity, $\left\langle C_{6}\right\rangle$ is expected to be useful for discriminating between solid-like and fluid-like condensed configurations (and in some cases between solid phases of different lattice structure). The third $\mathrm{OP}, D_{c c}{ }^{*}$, is introduced here for the first time and defined as the average pairwise distance between distinct clusters in the system normalized by the radius of a single particle. A cluster is defined as a subset of particles that are mutually connected by pairwise bonds. In the example problem studied here, a pair of colloidal particles is defined as bonded if they are close enough to experience the mutual attractive entropic interaction caused by the depletant particles as defined in section 2.1.1. The number of distinct clusters in an $n$ particle system may range from 1 to $n$. The pairwise distance between two clusters is defined as the smallest distance between any pair of particles, with the pair comprising one particle from each cluster. In the case of only one distinct cluster, $D_{c c}{ }^{*}$ is set to a value that corresponds to the maximum bonded length for a particle pair; this makes $D_{c c}{ }^{*}$ an essentially continuous function with a lower bound equal to the maximum bonded length.

Figure 3 illustrates four trajectories of the model system, each for a different value of the attraction strength, plotted in the three OPs. While the physical insight derived from these plots will become more apparent later, one can immediately see the relevant range of each OP and the stochastic nature of the trajectories in the OP variables.

\subsubsection{Constructing models in the OPs}

2.2.2.1. Low-dimensional Smoluchowski models. In past work ${ }^{51,52}$ we have constructed Smoluchowski models in the low-dimensional space of the chosen order-parameter set $\mathbf{X}$,

$$
\frac{\partial \rho(\mathbf{X}, t)}{\partial t}=\nabla \cdot e^{-\frac{F(\mathbf{X})}{k T}} \mathbf{D}(\mathbf{X}) \cdot \nabla e^{\frac{F(\mathbf{X})}{k T}} \rho(\mathbf{X}, t)
$$

where $\rho(\mathbf{X}, t)$ is the probability of the system being at location $\mathbf{X}$ in order parameter space at time $t, F(\mathbf{X})$ is the free energy landscape (FEL), and $\mathbf{D}(\mathbf{X})$ is the diffusivity landscape (DL). In writing such a model, we have assumed that the system undergoes a Markov process in the coarse-variable space. While we have not rigorously proven that this is true, it seems a reasonable starting point based on the fact that the colloidal system under consideration follows a Markov process in its basic coordinates $\mathbf{r}^{(N c)}$. Previously we have constructed the models of Eq. (9) by fitting the coefficients $F$ and $\mathbf{D}$ to direct BD simulations of the colloidal system using either a linear fit to many localized short trajectories ${ }^{54}$ or a fit to discretized transition rate matrices using Bayesian inference on longer trajectories. ${ }^{55}$ The FEL and DL provide an overview of the coarse thermodynamic and kinetic characteristics of the system.

To assess the quantitative accuracy of candidate low-dimensional Smoluchowski models, we compared probability propagator $\rho(\mathbf{X}, t)$ obtained by particle-scale BD simulations and low-dimensional Langevin dynamic (LDLD) simulations. The LDLD simulations are based on a Langevin equation given as 


$$
\mathbf{X}(t+\Delta t)=\mathbf{X}(t)-\mathbf{D}(\mathbf{X}) \cdot(k T)^{-1} \nabla F(\mathbf{X}) \Delta t+\nabla \cdot \mathbf{D}(\mathbf{X})+[2 \mathbf{D}(\mathbf{X}) \Delta t]^{1 / 2} \boldsymbol{\Gamma}(t),
$$

where the coefficients are the same as in Smoluchowski model, $\Delta t$ is the integration time step, and $\Gamma(t)$ is a Gaussian noise variable. An accurate Smoluchowski model will reproduce $\rho(\mathbf{X}, t)$ obtained from high-dimensional particle-scale BD simulations and LDLD.

\subsection{Process control}

Recent studies have demonstrated the success of the Markov decision process (MDP) in simulating and controlling the dynamics of stochastic and many-body systems. ${ }^{56,57}$ In our previous studies on colloidal assembly, we used MDP to achieve a highly ordered colloidal crystal in a Markov chain transition state model of the assembly process, ${ }^{25,58,59}$ using electric field ${ }^{25,58}$ and depletion force ${ }^{59}$ as the control input. Given these successes, in this paper, we construct a Markov decision process framework to study the control of the colloidal crystals to form a single crystalline state using the system osmotic pressure as the control variable, and implementing the control in a BD simulation.

As a version of dynamic programming, the Markov decision process is characterized by $\left\{K, S, A_{s}, P_{a}\right\}^{60,61}$ Here $K$ is the collection of the discrete time steps $k ; S$ is the state space of the system; and $A_{s}$ is the collection of all admissible control actions for state $s$. In this study, there are four states of the system that correspond to four different values of the attraction strength $\Pi$ defined by Eq. (6). Specifically, $A_{s}=\left\{\Pi_{1}{ }^{*}, \Pi_{2}{ }^{*}, \Pi_{3}{ }^{*}, \Pi_{4}{ }^{*}\right\}=\{1.05$, $1.11,1.30,1.90\} \times 10^{-7} \mathrm{~nm}^{-3}$ for all states $s \in S$, where $\Pi^{*}=\Pi /(k T) . P_{a}$ is the parameterized probability transition matrix for control action $a \in A_{s}$, which contains the probability $P_{a, i j}$ for the system to be in state $j$ after a time step of $\Delta t$ under control action $a$, given the current state of $i$.

The steps for constructing the transition matrices $P_{a}$ include: first, to identify and discretize the continuous system state into a finite number of discrete states; second, to approximate the transitions between the discrete states using samples of the full system. ${ }^{62}$ These samples may come from a dynamic simulation or from experimental measurements; here the BD simulation provides the samples. The DMap analysis (section

3.2) identifies the order parameters $R_{g}{ }^{*}$ and $D_{c c}{ }^{*}$ as an appropriate low-dimensional set of coarse variables for this system, so the system state is described by these variables with $R_{g}{ }^{*} \in\left[\begin{array}{ll}2.6 & 4.5\end{array}\right]$ and $D_{c c}{ }^{*} \in\left[\begin{array}{ll}2 & 8\end{array}\right]$ (ranges are defined by physical meaning). $R_{g}{ }^{*}$ is discretized into 96 evenly spaced intervals with an increment of $\Delta R_{g}{ }^{*}=0.02$, while $D_{c c}{ }^{*}$ is discretized into 61 evenly spaced intervals with increment $\Delta D_{c c}{ }^{*}=0.1$. These increments were selected by constructing transition rate models with different levels of discretization, to determine the optimal size to accurately represent the BD simulation. Independent Brownian dynamics simulations are conducted under each of the four constant $\Pi^{*}$ values to cover the effective state space that can be visited by the system. Based on the Brownian dynamics simulations, four independent Markov transition matrices $P_{a}$ are constructed, having a time step of $\Delta t=5 \mathrm{~s}$ for each of the four $\Pi^{*}$ values. 
We designed an infinite-horizon MDP to obtain the optimal control policy. ${ }^{60,}{ }^{61}$ To formulate the optimization problem, the objective function $J_{a}$ is defined as

$$
J_{a}(x)=E\left\{\sum_{k=0}^{\infty} \gamma^{k} R\left(x_{k}, a_{k}\right)\right\}
$$

where $E$ is the expectation operator needed to account for the stochasticity of the system. $a_{k} \in A_{s}$ is the control action at time step $k$, and $x_{k} \in S$ is the system state at time step $k . \gamma$ is the discount factor, which ensures the convergence of the optimal policy calculation, and is chosen as 0.99 .

$R\left(x_{k}, a_{k}\right)$ is the one-stage reward function when action $a$ is used, given the system state in $x$. The one-stage-reward function $R\left(x_{k}, a_{k}\right)$ is defined to drive the system simultaneously to a state with low $D_{c c}{ }^{*}$ and low $R_{g}{ }^{*}$ values, which corresponds to a single highly ordered crystal.

$$
R\left(x_{k}, a_{k}\right)=\frac{1}{R_{g}^{* 2}}+\frac{1}{D_{c c}^{* 2}}
$$

The optimal control policy is achieved by obtaining an input trajectory $a$ that maximizes the objective function $J_{a}$ over an infinite number of time steps, via dynamic programming; and the optimal policy $a^{*}(x) \in A$ is defined as

$$
a^{*}(x)=\arg \left\{\sup J_{a}(x)\right\}
$$

\section{Example problem}

\subsection{Model system}

We modeled a system of 32 colloidal particles interacting via the AO-depletion potential of Eq. (6). As mentioned in section 2.3, we employed four specific values of the attraction strength, $\Pi^{*}=\Pi / k T=1.05,1.11,1.3$, and 1.9 (all in units of $10^{-7} \mathrm{~nm}^{-3}$ ). This variation in $\Pi^{*}$ is sufficient to take the system from a state where it is purely fluid-like (1.05) to a state where it coexists in fluid- and solid-like states (1.11) and finally to a state where it is purely solid-like (1.3 and 1.9). At $\Pi^{*}=1.9$, the crystalline state is a deep energy well, effectively locking in the desired state. With respect to the other parts of the AO depletion potential, including the repulsive contribution, we used exactly the same mathematical forms and parameter values employed in previous work. ${ }^{51-53,63}$

\subsection{Diffusion map results}

In our earlier work ${ }^{52}$ we used trajectory data sets obtained from Brownian-dynamics simulations as the input to the DMap analysis. However, the time required to generate trajectories that sample all relevant regions of phase space, i.e. detailed particle coordinate space, can be prohibitively long. In this work, we have adopted the idea of 
integrating diffusion mapping with Monte Carlo umbrella sampling (MC-US) as proposed by Ferguson and coworkers. ${ }^{64}$ The umbrella sampling ensures a more complete coverage of the phase space, although the biased nature of the sampling must be accounted for when preparing the trajectory data set for the DMap analysis. We implemented the method as follows.

We chose $R_{g}{ }^{*}$ and $\left\langle C_{6}\right\rangle$ as the starting order parameter set. This pair of variables has been used in our past studies and serves as a reasonable starting point. We also employed the specific attraction strength value $\Pi^{*}=1.11$ because this value is known to produce the most complex free energy landscape, with local minima representing both fluid-like and crystalline-like states. ${ }^{52}$ We carried out umbrella sampling on a grid spacing of $(0.01$, $0.0625)$ in the OP space $\psi=\left(R_{g}{ }^{*},<C_{6}>\right)$. Partially overlapping windows with side length equal to 10 times the grid spacing were used to cover the OP space, with hard walls used to constrain the system within a window during an $\mathrm{MC}$ run. We ran 50 million Monte Carlo steps per window and, by stitching together the free energy profiles in adjacent windows, produced a free energy landscape (FEL), $F(\psi)$, at the resolution of the grid. From this process we also naturally obtained a large collection of detailed particle configurations, or snapshots, that span the range of $\psi$. We then associated with each snapshot a Boltzmann weight given by

$$
P\left(\psi_{i}\right)=\frac{e^{-\beta F\left(\psi_{i}\right)}}{\sum_{i=1}^{n} e^{-\beta F\left(\psi_{i}\right)}}
$$

We used two-dimensional interpolation on the grid to compute each $F\left(\psi_{i}\right)$. Under this probability distribution, we scaled up our data set using

$$
c_{i}=\operatorname{round}\left(\left[s P\left(\psi_{i}\right)\right]\right)
$$

where $s$ is a scaling factor and $c_{i}$ is the multiplicity of each snapshot $i$. In the data set, each snapshot was then replicated a number of times equal to $c_{i}\left(c_{i}=0\right.$ essentially removes that snapshot from the data set). This procedure transformed the inherently biased set of snapshots from MC-US into a set that is consistent with what an unbiased MC walk would have produced, assuming that the underlying choice of coarse variables provides a meaningful FEL. We set the scaling factor $s$ equal to $10^{6}$ and subsampled to generate a data set containing $\sim 10^{4}$ snapshots. We employed this pseudo-unbiased set of snapshots as the input to the DMap analysis. (We note that other procedures for generating the pseudo-unbiased data set, which would take advantage of the fact that the configurations within a given window already have the correct relative weighting, are possible.) We also analyzed two subsets of this master data set; one subset included only those snapshots located in the fluid-like well on the FEL, and the other included only those snapshots located in the crystalline-like well. This allowed us to understand certain features of the DMap results.

Figure 4 (a)-(c) shows the eigenvalue spectra for the fluid well, crystalline well, and twowell (master) data set, respectively. A spectral gap is observed after the first non-trivial eigenvalue in 4(a) and the first two non-trivial eigenvalues in 4 (b)-(c), which indicates a 
dimensionality of at most two. Figure 5 shows the data sets plotted in the coordinates of the first two non-trivial eigenvectors, i.e., in $v_{2}-v_{3}$ space. The data points are colored by the value of the order parameter $R_{g}{ }^{*}(\mathrm{a}, \mathrm{d}, \mathrm{g}),\left\langle C_{6}\right\rangle(\mathrm{b}, \mathrm{e}, \mathrm{h})$, or $D_{c c}{ }^{*}(\mathrm{c}, \mathrm{f}, \mathrm{i})$. The first row (ac) shows the data from the fluid well. The DMap coordinate $v_{2}$, corresponding to the single non-trivial eigenvalue in Fig. 5(a), correlates best with $R_{g}{ }^{*}$. The second row (d-f) shows the data from the solid well. The data points are clustered by both $R_{g}{ }^{*}$ and $\left\langle C_{6}\right\rangle$, but $D_{c c}{ }^{*}$ shows an excellent correlation with $v_{2}$ as indicated by the continuous change of color from left to right in Fig 5(f). The two-well (master) data set is shown in the third row (g-i). By comparing this row with the rows above, we see that the fluid data are localized near the vertex of the angle formed by the two branches of the crystalline data. As with the crystalline well data, $D_{c c}{ }^{*}$ correlates best with $v_{2}$. For the data sets that include the crystalline well, Figs. 5(d-i), we observe a symmetry about the $v_{3}=0$ axis. This symmetry suggests that this coordinate is capturing some inversion degrees of freedom, which are not of particular interest in this study. Overall, the DMap analysis leads us to conclude that the OP space $\psi=\left(R_{g}{ }^{*}, D_{c c}{ }^{*}\right)$ will provide a better coarse-grained representation than the originally chosen space $\left(R_{g}{ }^{*},\left\langle C_{6}>\right) . R_{g}{ }^{*}\right.$ correlates best with the only important coordinate in the fluid well. $D_{c c}{ }^{*}$ correlates best with the first important coordinate in the crystalline well, while the second important coordinate in this well seems to be associated with an inversion symmetry that is not of interest. Figures 5(d)-(e) and $5(\mathrm{~g})-(\mathrm{h})$ indicate redundancy of information contained by $R_{g}{ }^{*}$ and $\left\langle C_{6}\right\rangle$, suggesting that the latter variable may be replaced. The work below will therefore employ the lowdimensional variable set $\left(R_{g}{ }^{*}, D_{c c}{ }^{*}\right)$.

\subsection{Free energy and diffusivity landscapes}

By fitting the trajectories in the order parameter space from BD simulations, we obtained $F(\mathbf{X})$ and $\mathbf{D}(\mathbf{X})$ in the Smoluchowski model, as shown in Figs. 6 and 7. The FEL provides thermodynamic information on the likelihood of the system being in different regions of the order parameter space $\left(R_{g}{ }^{*}, D_{c c}{ }^{*}\right)$. The free energy differences capture the thermodynamic driving force that influences trajectory drift between different states. At $\Pi^{*}=1.05$, there is a global minimum located around $(4.2,4.9)$, which corresponds to fluidlike structures. As $\Pi^{*}$ increases to 1.11 , the appearance of the two wells located at (3.8, $4.9)$ and $(3.1,4.1)$ indicates the coexistence of fluid-like structure and solid-like structures. At higher $\Pi^{*}$, large FEL gradients drive fluid-like structures toward solid-like structure with low $D_{c c}{ }^{*}$ and low $R_{g}$. At regions around $(3.1,3.5)$, there exist a local minimum with $k T$ scale barrier prior to assembly trajectories entering the global minimum near $(3.0,2.2)$.

The $\mathbf{D}(\mathbf{X})$ shown in Fig. 7 characterize the drift and diffusion mediated by the free energy gradient. We present only the diagonal terms of the tensor $\mathbf{D}$, as the cross terms that characterize coupling between $D_{c c}{ }^{*}$ and $R_{g}$ are basically negligible. For each $\Pi^{*}$, the $D_{c c}{ }^{*} D_{c c}{ }^{*}$ component shows higher values at $D_{c c}{ }^{*} \sim 2-3$, which characterize the larger fluctuations due to particles attaching to or breaking apart from a cluster. For the highest $\Pi^{*}$ case, the region near $D_{c c}{ }^{*} \sim 3-5, R_{g}{ }^{*} \sim 3.0-3.2$ also has a higher diffusivity in the $D_{c c}{ }^{*} D_{c c}{ }^{*}$ component. For the $R_{g}{ }^{*} R_{g}{ }^{*}$ component, higher values appear at moderate extent of condensation $\left(R_{g}{ }^{*} \sim 3.2-4.0\right)$, where particle arrangement results in strong fluctuations in 
$R_{g_{*}}{ }^{*}$. Both the $D_{c c}{ }^{*} D_{c c}{ }^{*}$ and $R_{g}{ }^{*} R_{g}{ }^{*}$ components generally show decreasing values at lower $R_{g}{ }^{*}$ as the configuration is more condensed, with little room for the particle arrangement.

The validity of the low-dimensional Smoluchowski models are examined by comparing the probability propagator constructed from particle-scale BD simulation and orderparameter-scale LDLD simulations. As an example, Fig. 8 shows results for $\Pi^{*}=1.11$ and an observation time $\mathrm{t}=10 \mathrm{~s}$ for two starting points. The time scale is smaller than the global relaxation time (i.e., order $10^{3} \mathrm{~s}$ ) and larger than the Brownian time scale (i.e., order $10^{-1} \mathrm{~s}$ ) and is expected to capture transient assembly dynamics. Figure 8 shows good agreement between BD and LDLD simulations starting at $(4.4,5.2)$ and $(3.2,4.5)$, which are the locations of the two wells at $\Pi^{*}=1.11$. Similar comparisons for the fluid well at lower $\Pi^{*}$ and similar FEL at higher $\Pi^{*}$ confirm the high-dimensional particle scale model can be well approximated by low dimensional order parameter based dynamic models.

\subsection{Process control using MDP}

Following the methods described in section 2.3, we constructed four transition matrices, one for each of the four osmotic pressure values $\Pi^{*}$ in the action space. To evaluate the accuracy of these transition matrices, Fig. 9 shows the 1000-realization averaged Markov chain Monte Carlo simulation based on sampling of the transition matrices, compared to 1000-realization averaged Brownian dynamics simulations. All these simulations are run under a constant $\Pi^{*}$ value, i.e., without control.

In Fig. 9, 'BDUnCon' denotes the uncontrolled Brownian-dynamics simulation and 'MCUnCon' denotes the uncontrolled Markov chain Monte Carlo simulation. All the simulations started from the same fluid-like initial state with $R_{g}{ }^{*}=4.4$ and $D_{c c}{ }^{*}=5.15$, and were simulated for 500 seconds. According to this comparison, the transition matrices $P_{a}$ are able to approximate the BD simulation qualitatively and with reasonable quantitative accuracy, capturing the differences between the four osmotic pressures. Under high $\Pi^{*}$ values $\left(\Pi_{3}{ }^{*}\right.$ and $\left.\Pi_{4}{ }^{*}\right)$, the system is able to reach a more crystalline final state (given the same amount of simulation time), indicated by lower $R_{g}{ }^{*}$ and $D_{c c}{ }^{*}$ values. Meanwhile, the system under $\Pi_{3}{ }^{*}$ can achieve greater condensation indicated by $R_{g}{ }^{*}$, compared to $\Pi_{4}{ }^{*}$. On the other hand, the system is able to achieve a lower $D_{c c}{ }^{*}$ value under $\Pi_{4}{ }^{*}$ relative to $\Pi_{3}{ }^{*}$. Given these observations, some combination of $\Pi_{3}{ }^{*}$ and $\Pi_{4}{ }^{*}$ might be desirable, in order to accomplish the goals of low $R_{g}{ }^{*}$ and $D_{c c}{ }^{*}$.

Given the transition matrix models, we constructed a time-independent optimal control policy with dynamic programming using the policy iteration algorithm. This twodimensional control policy is shown in Fig. 10 as a lookup table. The numbers in the color bar stand for the control action $\Pi^{*}$ values, increasing from $\Pi_{1}{ }^{*}$ to $\Pi_{4}{ }^{*}$ accordingly. Given its time-independent property, with the current $R_{g}{ }^{*}$ and $D_{c c}{ }^{*}$ values known, the corresponding control action can be taken directly from this table. With approximately 10,000 discrete states, the policy calculation can be achieved in only a few minutes.

According to Fig. 10, the optimal control policy chooses the high $\Pi^{*}$ value, $\Pi_{3}{ }^{*}$ and $\Pi_{4}{ }^{*}$, in most of the order-parameter state space. When the system is close to the global 
minimum point around $R_{g}{ }^{*}=2.8$ and $D_{c c}{ }^{*}=2.2$, indicated by the FELs, $\Pi_{4}{ }^{*}$ control is used in order to lock the system in the global minimum state. On top of the control policy is plotted a single controlled BD simulation result, starting from a fluid-like state of $R_{g}{ }^{*}=3.78$ and $D_{c c}{ }^{*}=5.0$. By switching between $\Pi_{3}{ }^{*}$ and $\Pi_{4}{ }^{*}$, the system is able to reach a final state of $R_{g}{ }^{*}=2.9$ and $D_{c c}{ }^{*}=2.2$.

Moreover, the effectiveness of the control policy is evaluated by applying it to both the Markov chain transition matrix model and the BD simulation. A comparison of 250realization-averaged order parameter simulation results with and without control in Brownian dynamics is given in Fig. 11.

In Fig. 11, the trajectory labeled as 'BDCon' stands for the 250-realization-averaged BD simulations with control. Trajectories labeled preceded with 'BDPi' are 250-realization averaged Brownian dynamics simulation under constant $\Pi^{*}$ values. All these trajectories are simulated for 500 seconds, starting from the same initial fluid-like state of $R_{g}{ }^{*}=3.78$ and $D_{c c}{ }^{*}=5.0$. Fig. 11(a) shows the averaged trajectories in the order-parameter phase plane. Inspection of the controlled trajectory reveals a decrease in the final $D_{c c}{ }^{*}$ value, compared to that under $\Pi_{3}{ }^{*}$, as well as a lower $R_{g}{ }^{*}$ value compared to that under $\Pi_{4}{ }^{*}$. Fig. 11(b) shows the same results as in Fig. 11(a), but now as a function of time. In all three cases the system responds on approximately the same timescale, but achieves different long-term states.

\section{Conclusion}

Application of feedback control in colloidal assembly is challenging, due to the nonlinear, stochastic, and high-dimensional dynamics, as well as the difficulty of realtime measurement. However, recent advantages in computation and in situ sensing make optimal model-based feedback control feasible. Reduced-order state representations and dynamic models link naturally to the framework of the Markov decision process, providing the optimal policy in a table-lookup form that requires virtually no online computation. Application of this approach to a Brownian Dynamics simulation of depletion-force mediated self-assembly demonstrates the feasibility of the approach, as well as the ability of a time-varying input to drive the system to a more desirable crystalline state.

\section{Acknowledgments}

M.A.B., D.M.F., M.A.G. and B.S. acknowledge financial support by the National Science Foundation through a set of collaborative Cyber Enabled Discovery and Innovation Grants (Awards No. CMMI-1124648, CMMI-1125188, CMMI-1124678, and CMMI1261938). D.M. and R.M.S. acknowledge support by the U.S. Department of Energy, Office of Basic Energy Sciences, Division of Materials Sciences and Engineering, under Award No. DE-FG02-07ER46407.

\section{Figure Captions}


Figure 1. An overview of how colloidal assembly can be controlled by real-time manipulation of interaction forces.

Figure 2. (a) Schematic of charged $2.34 \mu \mathrm{m} \mathrm{SiO}{ }_{2}$ colloidal particles with particle-wall or particle-particle surface separation, $h$, experiencing depletion attraction with each other and a glass microscope slide due to the exclusion of spherical depletants (yellow) with radius, $L$. Depletants can approach the $\mathrm{SiO}_{2}$ surfaces within a distance, $\Delta_{\mathrm{EV}}$. Depletants can approach each other within a distance, $\Delta_{\mathrm{OP}}{ }^{65}$ (b) Particle-wall potential energy profiles obtained using Total Internal Reflection Microscopy. ${ }^{20}$

Figure 3. Four different representative dynamic trajectories of the model colloidal system. Each trajectory is plotted in terms of the three coarse variables considered in this study: radius of gyration $R_{g}{ }^{*}$, crystallinity metric $\left\langle C_{6}\right\rangle$, and average cluster-to-cluster distance $D_{c c}{ }^{*}$. The trajectories are for different values of osmotic pressure $\Pi^{*}=1.05(\mathbf{a})$, 1.11 (b), 1.30 (c), and 1.90 (d), with $\Pi^{*}$ defined later in the text.

Figure 4. Eigenvalue spectra for data sets comprising (a) fluid, (b) crystal and (c) both fluid and crystal states. For the fluid data set there is a spectral gap after the first nontrivial eigenvalue, but for the crystal and combined data sets there is a spectral gap after the first two non-trivial eigenvalues. These findings indicate a requirement of at most two dimensions in the low-dimensional model.

Figure 5. Diffusion map representation of the three data sets described in the text, in the space of the first two eigenvectors. The top row $(\mathbf{a}, \mathbf{b}, \mathbf{c})$ is for the fluid data set, the middle row (d,e,f) is for the crystal data set, and the bottom row $(\mathbf{g , h}, \mathbf{i})$ is for the combined data set. In the first column (a,d,g,) the data points are colored by $R_{g}{ }^{*}$, in the second column $(\mathbf{b}, \mathbf{e}, \mathbf{h})$ the data points are colored by $\left\langle C_{6}\right\rangle$, and in the third column $(\mathbf{c}, \mathbf{f}, \mathbf{i})$ the data points are colored by $D_{c c}{ }^{*}$.

Figure 6. Free energy landscapes $F(\mathbf{X}) / k T$ as a function of $D_{c c}{ }^{*}$ and $R_{g}{ }^{*}$ obtained by fitting trajectories to the Smoluchowski equation at $\Pi^{*}=1.05$ (a), 1.11 (b), 1.30 (c), and 1.90 (d).

Figure 7. Diagonal components $D_{D c c^{*} D c c^{*}}$ (upper row) and $D_{R g^{*} R g^{*}}$ (lower row) of the diffusivity landscape, in units of $\mathrm{s}^{-1}$, obtained by fitting trajectories to Smoluchowski equation at $\Pi^{*}=1.05(\mathbf{a}, \mathbf{e}), 1.11(\mathbf{b}, \mathbf{f}), 1.30(\mathbf{c}, \mathbf{g})$, and $1.90(\mathbf{d}, \mathbf{h})$.

Figure 8. Probability propagator $\rho(\mathbf{X}, t)$ obtained from BD and LDLD at $\Pi^{*}=1.11$ with starting point $(4.4,5.2)$ (a) BD (b) LDLD and starting point $(3.2,4.5)$ (c) BD (d) LDLD with observation time $10 \mathrm{~s}$.

Figure 9. Comparison between averaged $\mathrm{MCMC}$ and BD simulation order parameter trajectories for different constant input $\Pi^{*}$ values from a system without feedback control. 
Figure 10. Optimal control policy computed based on Markov chain model using dynamic programming.

Figure 11. Comparison between 500-second 250-realization-averaged order parameter trajectories with and without the control policy.

\section{REFERENCES}

1. O. D. Velev and S. Gupta, Advanced Materials 21 (19), 1897-1905 (2009).

2. M. Grzelczak, J. Vermant, E. M. Furst and L. M. Liz-Marzán, ACS Nano 4 (7), 3591-3605 (2010).

3. S. C. Glotzer and M. J. Solomon, Nat Mater 6 (7), 557-562 (2007).

4. D. E. Chang and N. Petit, International Journal of Robust and Nonlinear Control 15 (16), 769--784 (2005).

5. B. Edwards, N. Engheta and S. Evoy, Journal of Applied Physics 102 (2), 024913--024915 (2007).

6. M. P. Melnyk and D. E. Chang, Asian Journal of Control, n/a (2006).

7. G. J. Shah, A. T. Ohta, E. P. Y. Chiou, M. C. Wu and C.-J. C. J. Kim, Lab on a Chip 9 (12), 1732-1739.

8. J. K. Fisher, J. Cribb, K. V. Desai, L. Vicci, B. Wilde, K. Keller, R. M. Taylor, J. Haase, K. Bloom, E. T. O Brien and R. Superfine, Review of Scientific Instruments 77 (2), 023702 (2006).

9. C. Gosse and V. Croquette, Biophysical Journal 82 (6) (2002).

10. M. P. Kummer, J. J. Abbott, B. E. Kratochvil, R. Borer, A. Sengul and B. J. Nelson, Robotics, IEEE Transactions on 26 (6), 1006-1017 (2010).

11. H. Lee, Y. Liu, R. M. Westervelt and D. Ham, IEEE Journal of Solid-State Circuits 41 (6), 1471-- 1480 (2006).

12. M. Armani, S. Chaudhary, R. Probst and B. Shapiro, in uTAS (Malmo, Sweden, 2004), pp. 26-30.

13. M. Armani, S. Chaudhary, R. Probst and B. Shapiro, Journal of Micro-ElectroMechanical Systems 15 (4), 945-956 (2006).

14. A. E. Cohen and W. E. Moerner, Optics Express 16 (10), 6941--6956 (2008).

15. M. D. Curtis, G. J. Sheard and A. Fouras, Lab on a Chip (2011).

16. R. Probst, Z. Cummins, C. Ropp, E. Waks and B. Shapiro, IEEE Control Systems Magazine 32 (2), 26--53 (2012).

17. M. Tanyeri, M. Ranka, N. Sittipolkul and C. M. Schroeder, Lab on a Chip (2011).

18. P. L. Biancaniello, A. J. Kim and J. C. Crocker, Phys. Rev. Lett. 94, 058302 (2005).

19. F. Soyka, O. Zvyagolskaya, C. Hertlein, L. Helden and C. Bechinger, Physical Review Letters 101 (20), 208301 (2008).

20. G. E. Fernandes, D. J. Beltran-Villegas and M. A. Bevan, Langmuir 24, 1077610785 (2008).

21. G. E. Fernandes, D. J. Beltran-Villegas and M. A. Bevan, J. Chem. Phys. 131, 134705 (2009). 
22. J. J. Juarez and M. A. Bevan, Advanced Functional Materials 22 (18), 3833-3839 (2012).

23. J. J. Juarez, S. E. Feicht and M. A. Bevan, Soft Matter 8 (1), 94-103 (2012).

24. J. J. Juarez, P. P. Mathai, J. A. Liddle and M. A. Bevan, Lab on a Chip 12 (20), 4063-4070 (2012).

25. Y. Xue, D. J. Beltran-Villegas, X. Tang and M. A. Bevan, IEEE Transactions on Control Systems Technology published on the web, doi:10.1109/TCST.2013.2296700 (2014).

26. C. F. Zukoski, Chemical Engineering Science 50 (24), 4073-4079 (1995).

27. D. A. McQuarrie, Statistical Mechanics. (HarperCollins, New York, 1976).

28. W. B. Russel, D. A. Saville and W. R. Schowalter, Colloidal Dispersions. (Cambridge University Press, New York, 1989).

29. D. Frenkel, Physica A 313 (1-2), 1-31 (2002).

30. L. Onsager, Ann Ny Acad Sci 51 (4), 627-659 (1949).

31. L. Belloni, J Phys-Condens Mat 12 (46), R549-R587 (2000).

32. T. L. Hill, An Introduction to Statistical Thermodynamics. (Dover, New York, 1986).

33. W. G. Mcmillan and J. E. Mayer, J Chem Phys 13 (7), 276-305 (1945).

34. S. Asakura and F. Oosawa, J Chem Phys 22 (7), 1255-1256 (1954).

35. G. E. Fernandes, D. J. Beltran-Villegas and M. A. Bevan, Langmuir 24 (19), 10776-10785 (2008).

36. G. E. Fernandes, D. J. Beltran-Villegas and M. A. Bevan, J Chem Phys 131 (13) (2009).

37. D. C. Prieve, Adv. Colloid Interface Sci. 82 (1-3), 93-125 (1999).

38. M. A. Bevan and S. L. Eichmann, Curr. Opin. Colloid Interface Sci. 16 (2), 149157 (2011).

39. J. Israelachvili, Y. Min, M. Akbulut, A. Alig, G. Carver, W. Greene, K. Kristiansen, E. Meyer, N. Pesika, K. Rosenberg and H. Zeng, Rep. Prog. Phys. 73 (3), 16 (2010).

40. W. A. Ducker, T. J. Senden and R. M. Pashley, Nature 353 (6341), 239-241 (1991).

41. D. L. Ermak and J. A. Mccammon, J Chem Phys 69 (4), 1352-1360 (1978).

42. T. J. Murphy and J. L. Aguirre, J Chem Phys 57 (5), 2098-\& (1972).

43. J. F. Brady and G. Bossis, Annual Review of Fluid Mechanics 20, 111-157 (1988).

44. D. J. Beltran-Villegas, R. M. Sehgal, D. Maroudas, D. M. Ford and M. A. Bevan, J Chem Phys 132 (4) (2010).

45. H.-J. Wu, T. O. Pangburn, R. E. Beckham and M. A. Bevan, Langmuir 21 (22), 9879-9888 (2005).

46. T. O. Pangburn and M. A. Bevan, J. Chem. Phys. 123, 174904 (2005).

47. T. O. Pangburn and M. A. Bevan, J. Chem. Phys. 124, 054712 (2006).

48. R. R. Coifman, S. Lafon, A. B. Lee, M. Maggioni, B. Nadler, F. Warner and S. W. Zucker, P Natl Acad Sci USA 102 (21), 7426-7431 (2005).

49. A. L. Ferguson, A. Z. Panagiotopoulos, I. G. Kevrekidis and P. G. Debenedetti, Chem Phys Lett 509 (1-3), 1-11 (2011). 
50. B. Nadler, S. Lafon, R. R. Coifman and I. G. Kevrekidis, Appl Comput Harmon A 21 (1), 113-127 (2006).

51. D. J. Beltran-Villegas, R. M. Sehgal, D. Maroudas, D. M. Ford and M. A. Bevan, J Chem Phys 135 (15) (2011).

52. D. J. Beltran-Villegas, R. M. Sehgal, D. Maroudas, D. M. Ford and M. A. Bevan, J Chem Phys 137 (13) (2012).

53. R. M. Sehgal, J. G. Cogan, D. M. Ford and D. Maroudas, Appl Phys Lett 102 (20) (2013).

54. D. I. Kopelevich, A. Z. Panagiotopoulos and I. G. Kevrekidis, J Chem Phys 122 (4), - (2005).

55. G. Hummer, New J Phys 7, - (2005).

56. A. Banerjee, A. Pomerance, W. Losert and S. Gupta, IEEE Transactions on Automation Science and Engineering 7, 218-227 (2010).

57. Y. Xue and M. A. Grover, in 2011 American Control Conference (San Francisco, CA, 2011), pp. 3269-3274.

58. X. Tang, Y. Yang, M. A. Bevan and M. A. Grover, in 2014 American Control Conference (Portland, OR, 2014), pp. accepted.

59. Y. Xue, D. Beltran-Villegas, M. A. Bevan and M. A. Grover, in 2013 American Control Conference (Washington, D. C., 2013), pp. 3334-3339.

60. D. P. Bertsekas, Dynamic Programming and Optimal Control, Volume 2. (Athena Scientific, Belmont, MA, 2012).

61. M. L. Puterman, Markov Decision Processes Discrete Stochastic Dynamic Programming. (Wiley-Interscience, Hoboken, NJ, 2005).

62. J.-H. Prinz, H. Wu, M. Sarich, B. Keller, M. Senne, M. Held, J. D. Chodera, C. Schutte and F. Noe, The Journal of Chemical Physics 134, 174105 (2011).

63. D. J. Beltran-Villegas and M. A. Bevan, Soft Matter 7 (7), 3280-3285 (2011).

64. A. L. Ferguson, A. Z. Panagiotopoulos, P. G. Debenedetti and I. G. Kevrekidis, J Chem Phys 134 (13) (2011).

65. T. D. Edwards and M. A. Bevan, Langmuir 28 (39), 13816-13823 (2012). 

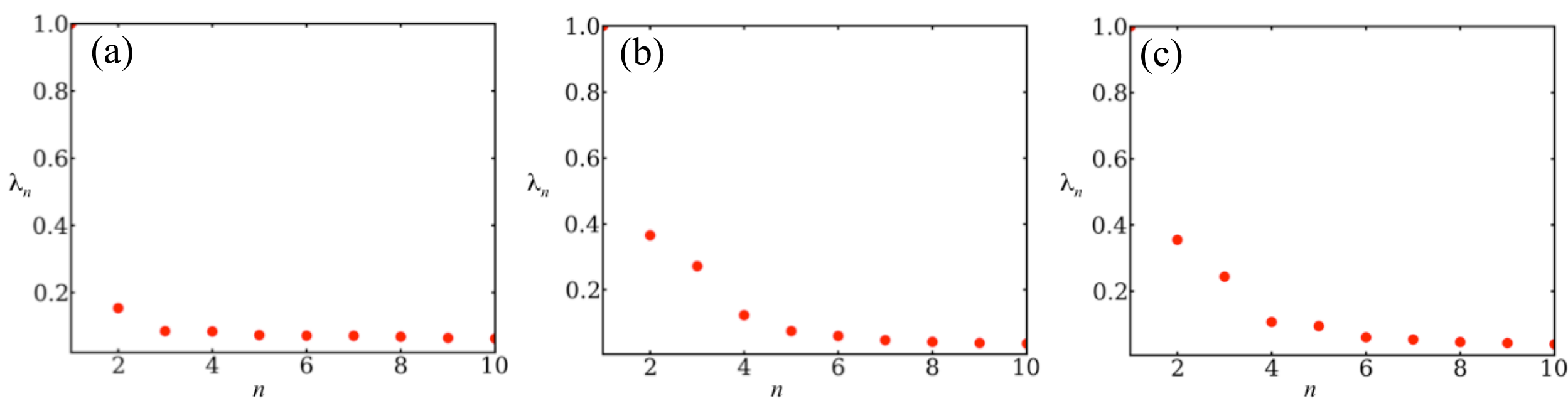

Figure 4 


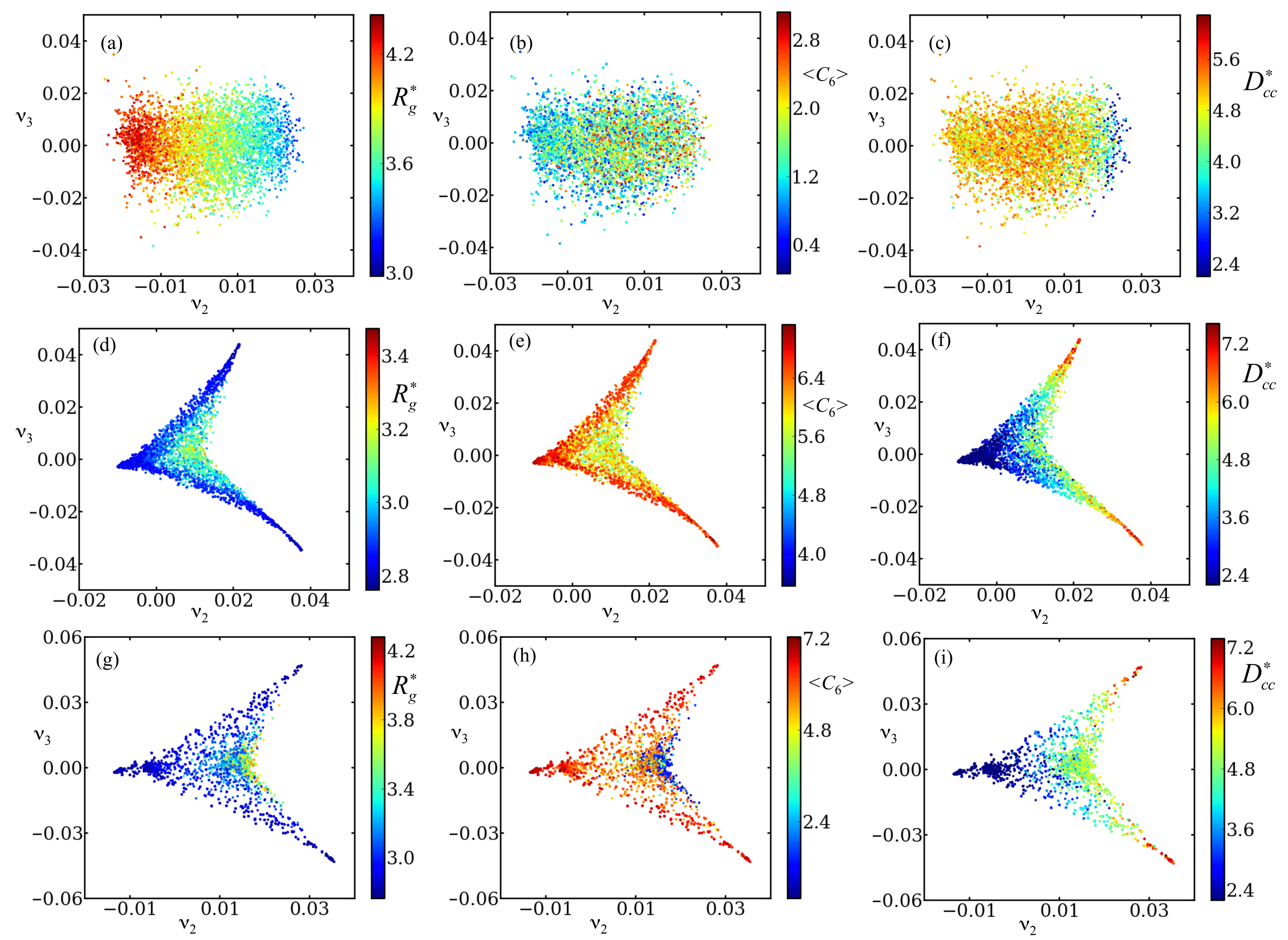

\section{Figure 5}



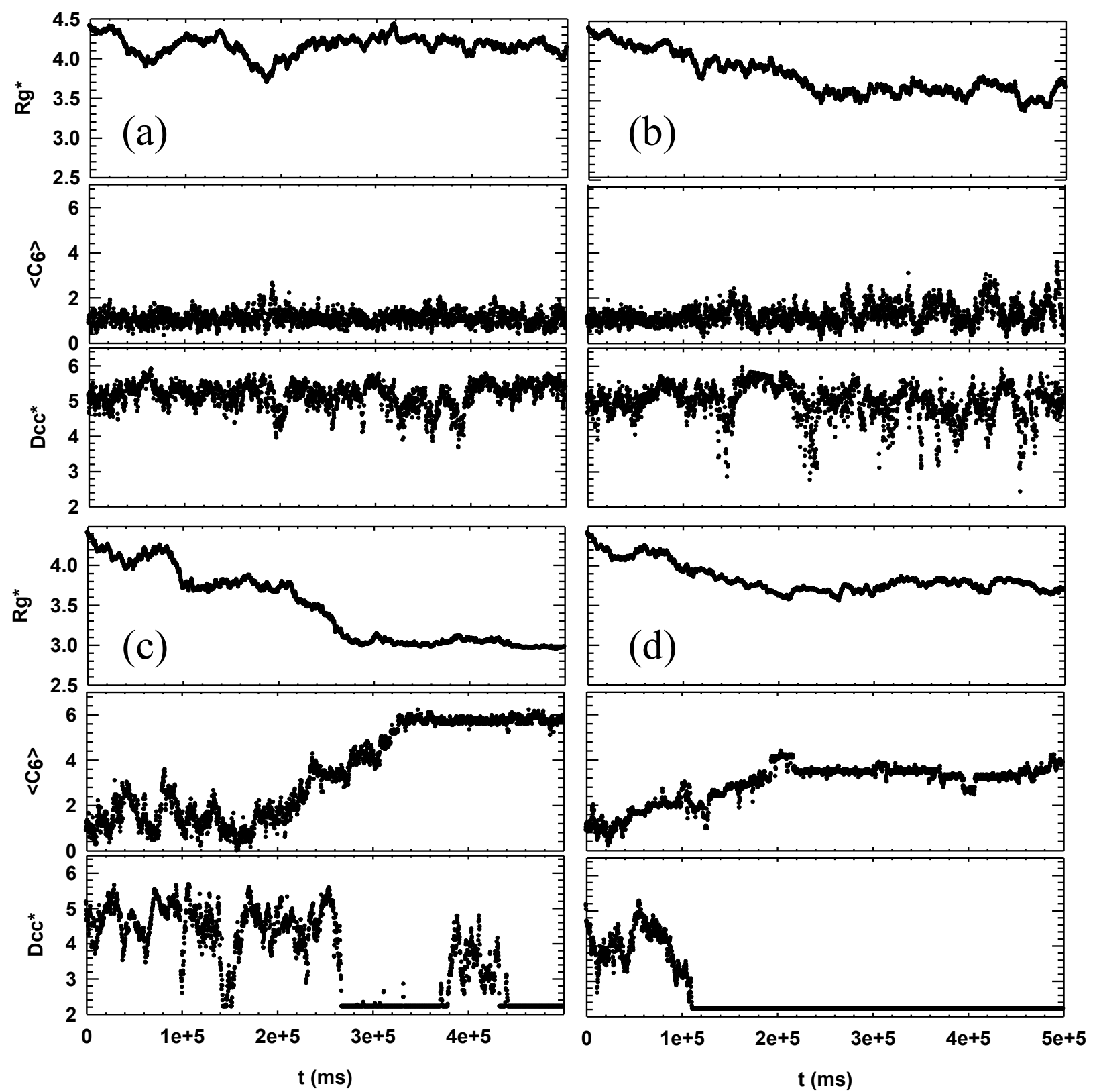

Figure 3 


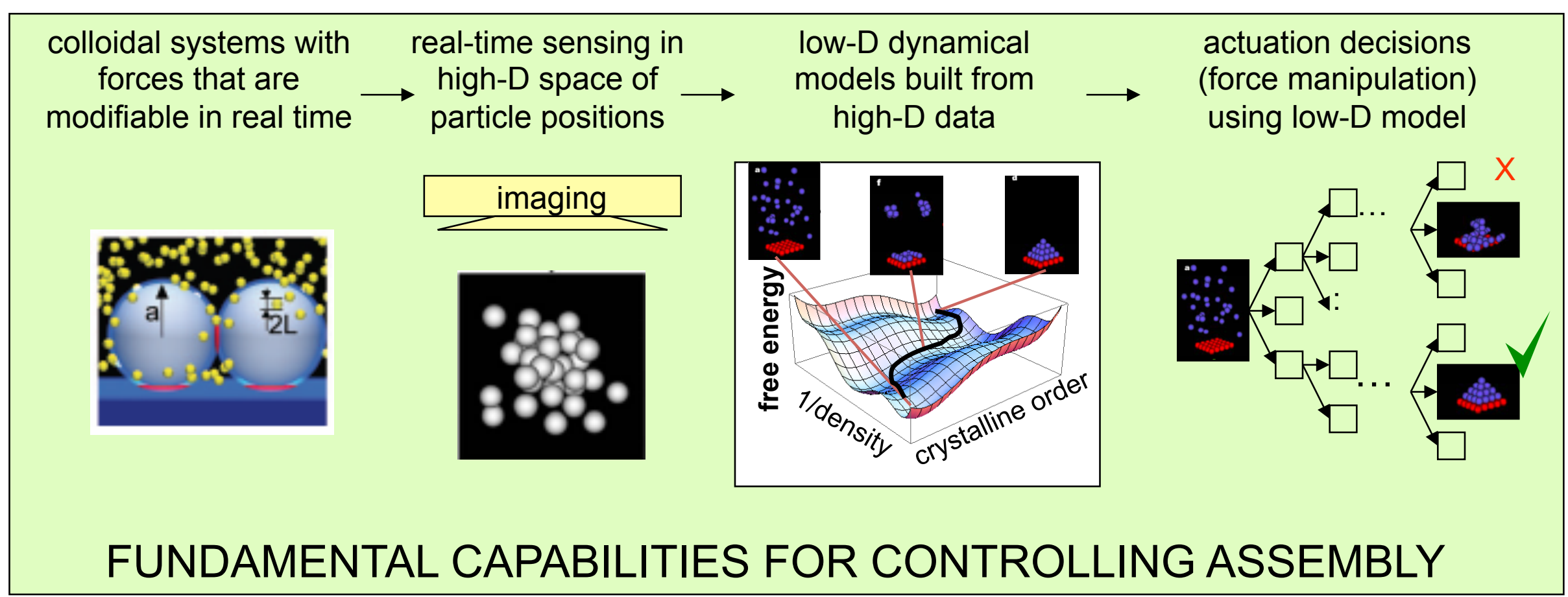

Figure 1 


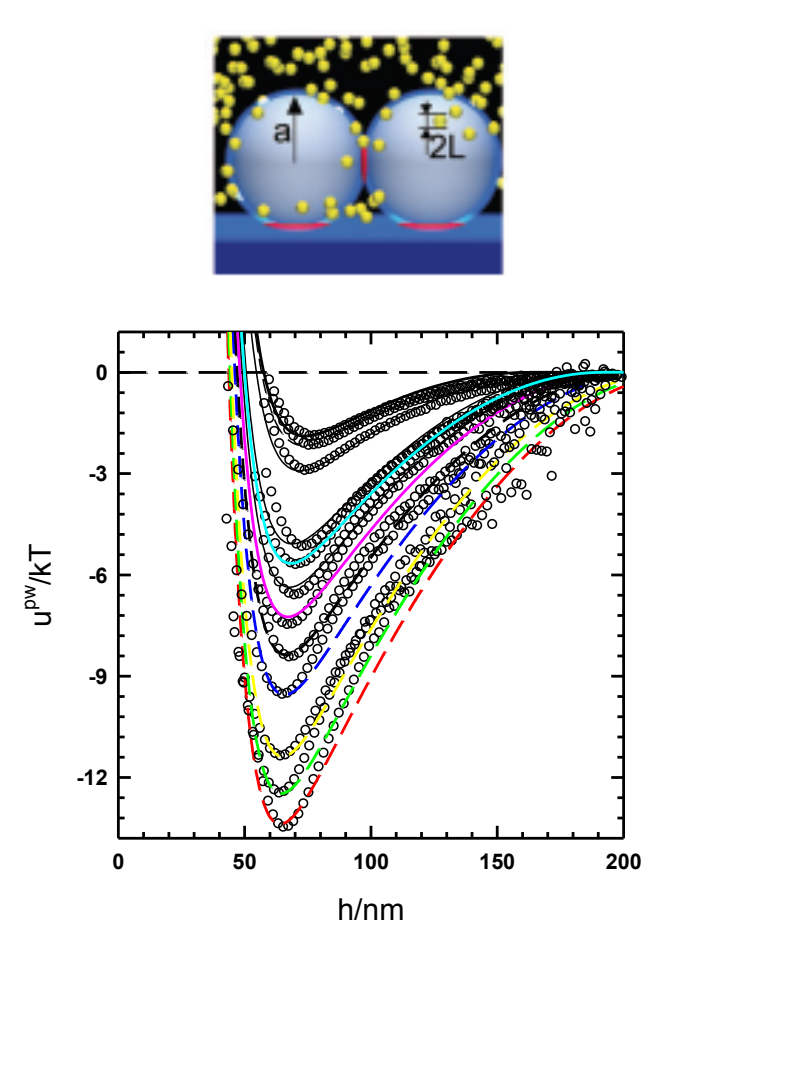

Figure 2

Figure 2

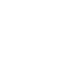

Figure 2

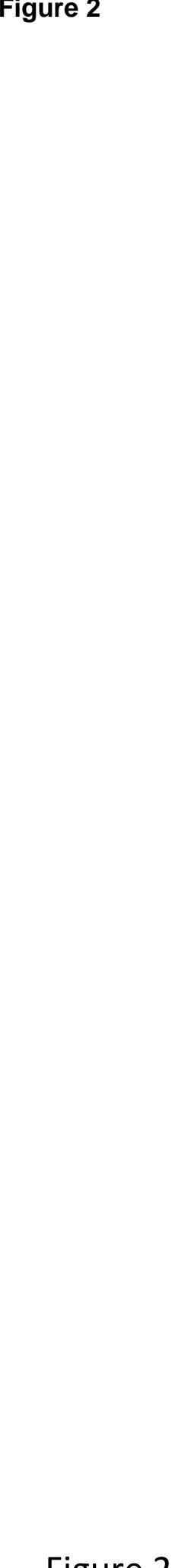

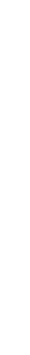

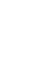
政

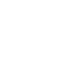
( (n) 政 (n) (n) (n) (n) (n)

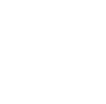

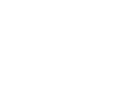

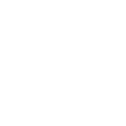
更

h/nm

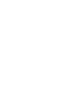

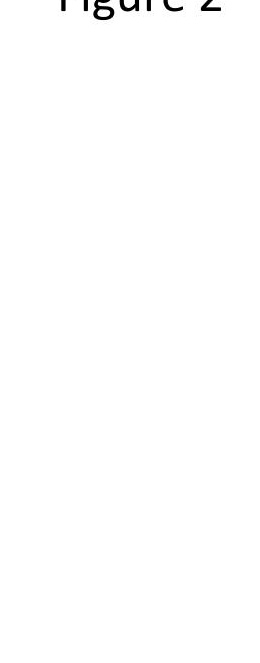

( 

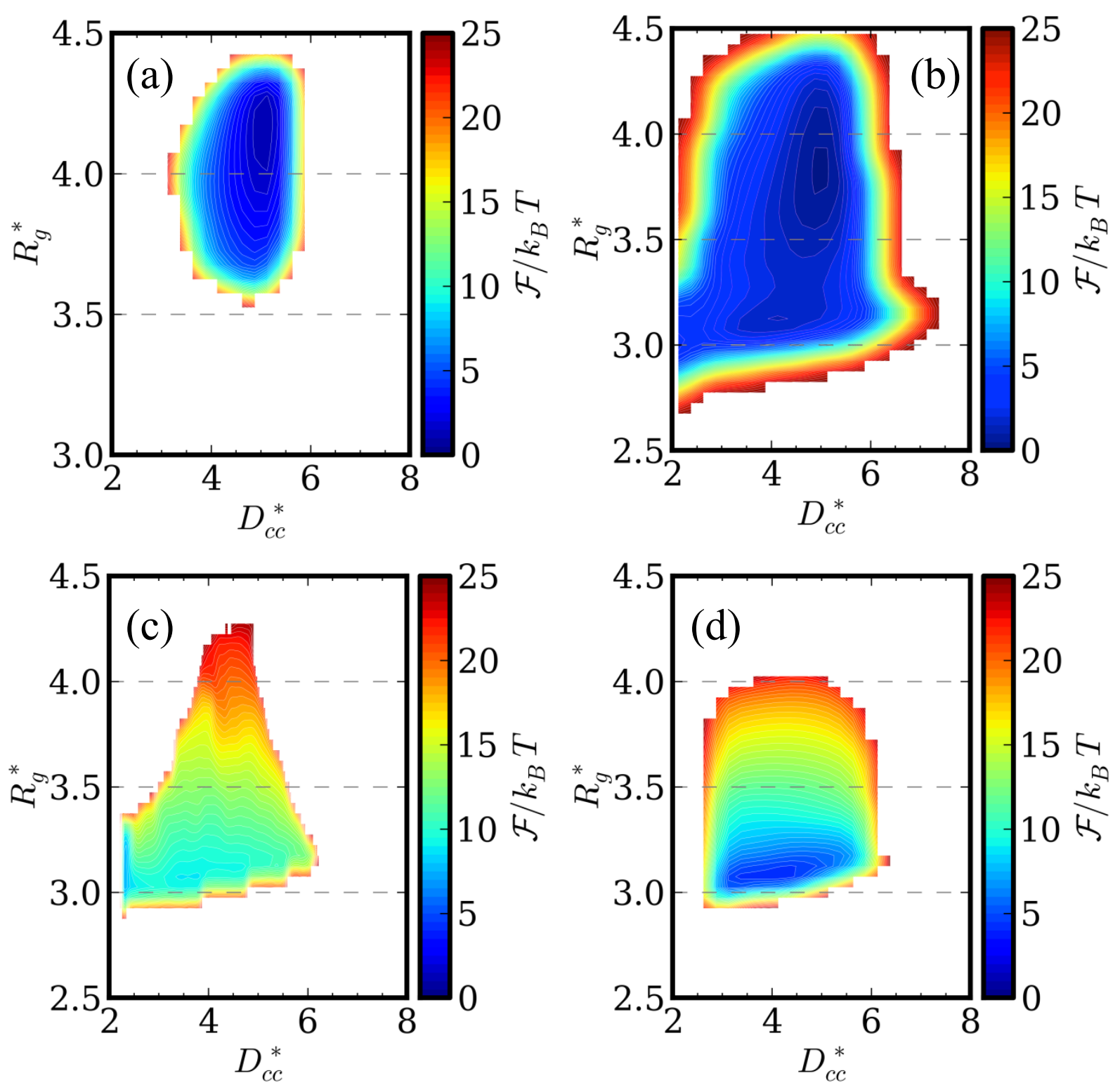

Figure 6 

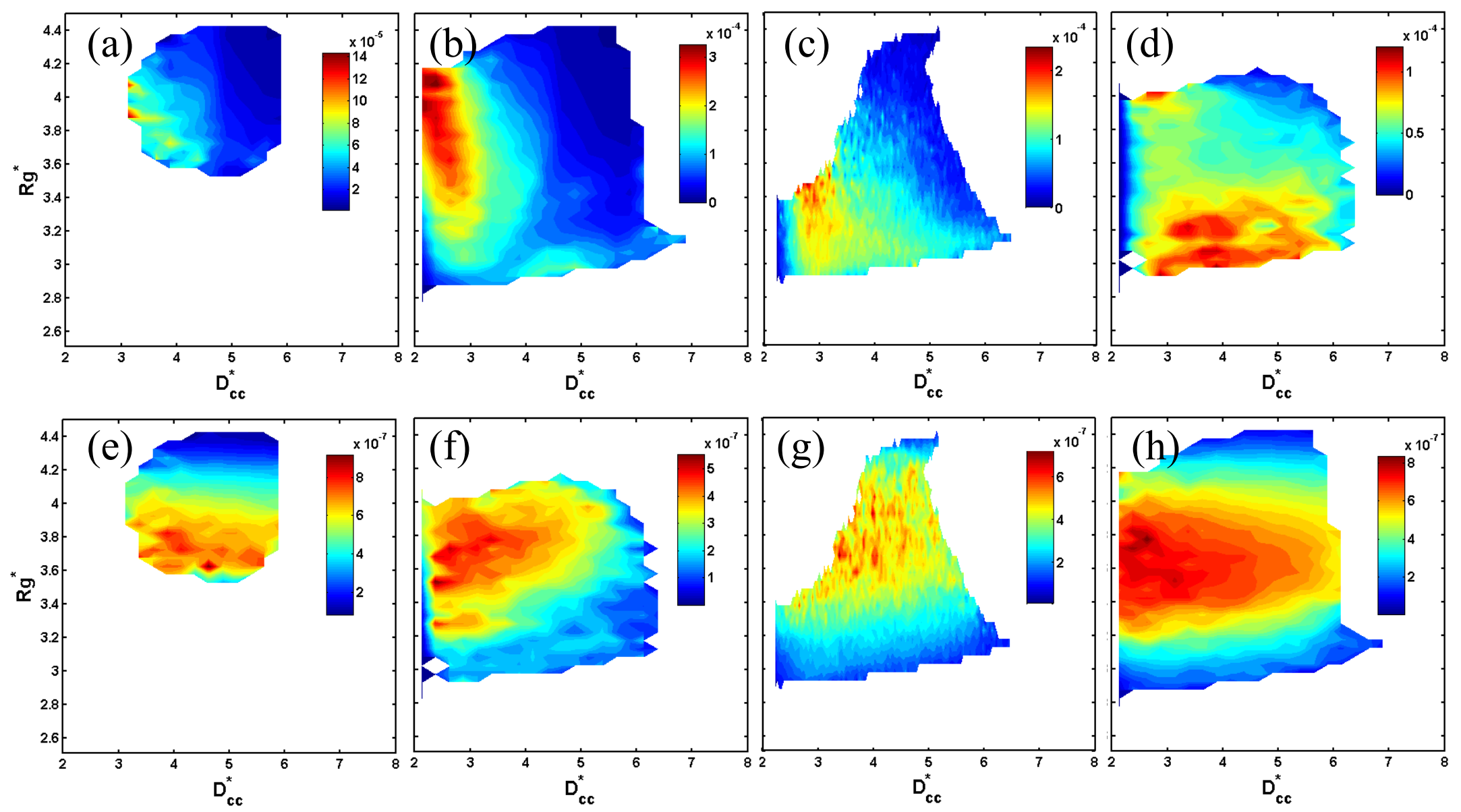

Figure 7 
Figure 8

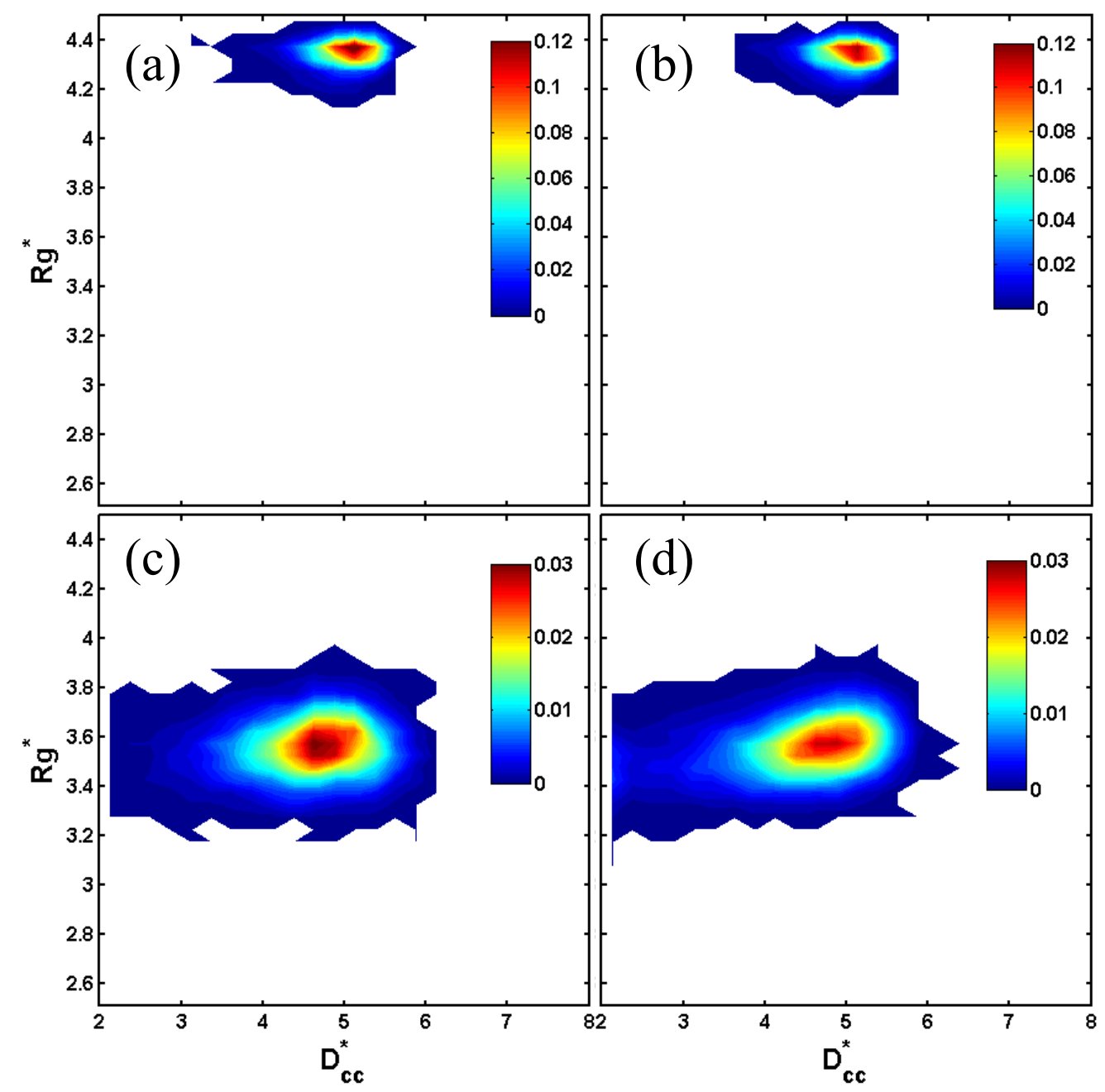

Figure 8 
Figure 9

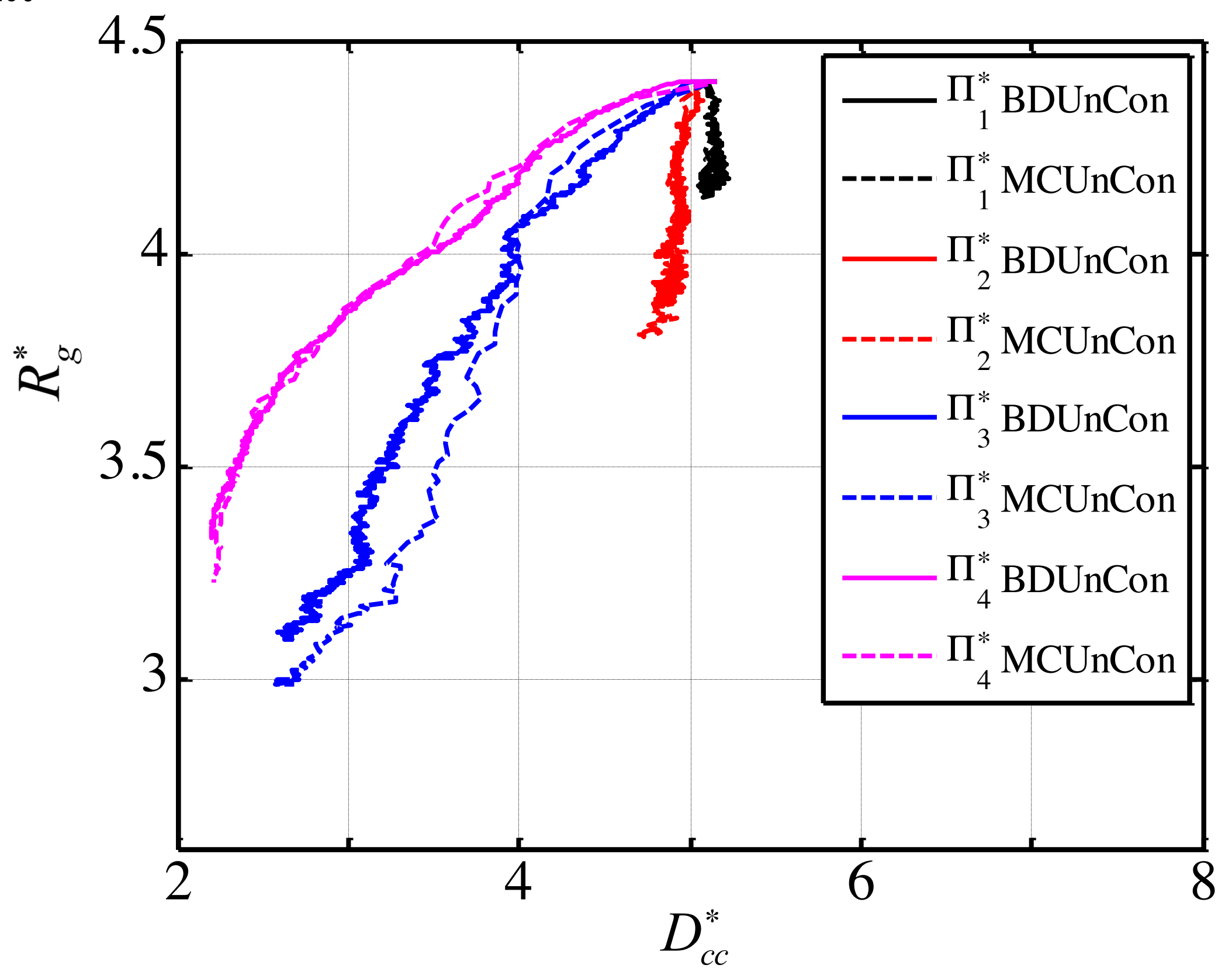

Figure 9 
Figure 104

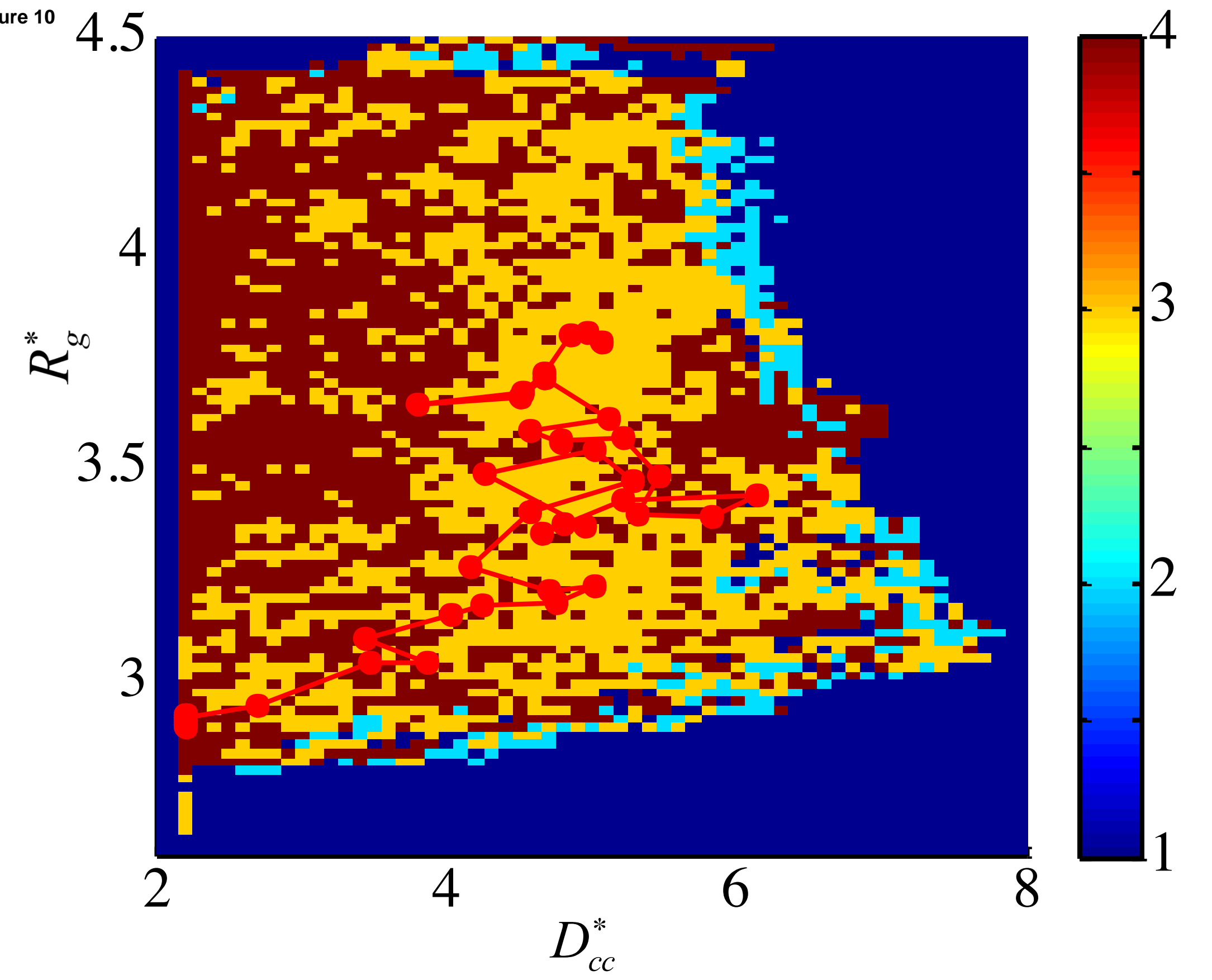

Figure 10 
